\title{
Tra «buona sociabilità» e avanguardia patriottica. Censura teatrale e costruzione del consenso in età risorgimentale
}

\author{
Nicola Gabriele
}

La censura non ha storia. Non perché trascende la storia, ma per il motivo opposto: non esiste infatti un fenomeno storico che possa dirsene esente. La censura, allora, può essere oggetto di studio in sé, poiché essa possiede una vita propria a prescindere dal momento nel quale, e sul quale, agisce. Multiforme, brutalmente esplicita oppure insidiosamente silente, imposta da apparati ideologici o filtrata attraverso l'interiorità soggettiva, la censura agisce costantemente e continuamente e anzi, proprio come l'ideologia, costituisce un elemento strutturale ineliminabile che investe la comunicazione umana in generale e le forme dell'espressione artistica in particolare ${ }^{\mathrm{I}}$.

L'analisi offerta da Annalisa Goldoni e Carlo Martinez nel loro volume Teatro e censura è preziosa per introdurre una riflessione sul rapporto tra queste due forme di esercizio dell'ingegno e dell'intelletto.

Rappresentazione teatrale e attività censoria sono due fenomeni culturali che nell'Europa d'Ancien régime e nell'Italia risorgimentale assumono una valenza ideologica non solo se analizzati singolarmente, ma ancor più quando li si coniuga e li si pone in una reciproca relazione. Per esaminarne la complementarietà è efficace un approccio che tenti di analizzarne alcuni caratteri specifici, non solo per coglierne eventuali convergenze, ma anche per elaborare una vera e propria prospettiva storiografica e delineare alcuni modelli interpretativi.

Su questo tema sono oggi imprescindibili gli studi condotti da Carlotta Sorba e Irene Piazzoni e prima ancora da Giovanni Morelli e Massimo Mila capaci di tracciare il solco di successivi approfondimenti ${ }^{2}$. Superato un approccio aneddotico, la censura teatrale in età risorgimentale meriterebbe di essere studiata e analizzata contestualmente a quella della stampa, sia perché in prevalenza i regolamenti della prima erano inclusi nei più generali ordinamenti censori che regolavano la seconda, sia perché la progressiva acquisizione di una libertà di stampa e di espressione a partire dal I848 determinò una nuova e originale geografia del teatro italiano. Studiare la censura teatrale nell'età della Restaurazione significa indagare il ruolo che il teatro esercitò sulla società italiana di quel periodo. Le strategie di controllo su

I. Teatro e censura, a cura di A. Goldoni e C. Martinez, Napoli, Liguori, 2004, p. I.

2. Sul ruolo del teatro nel Risorgimento cfr. Opera and Nation in Nineteenth-Century Italy, in «Journal of modern italian studies», vol. I7, n. 4, 20I2, pp. 393-502. 
ogni tipo di espressione del pensiero sono indice delle capacità che quelle forme di comunicazione avevano di incidere sulla società e di orientarla. Per questa ragione la censura seppe assumere caratteri e identità non sempre facilmente identificabili; l'abilità dei governi e delle cancellerie di quell'epoca fu di saper adottare forme di controllo e di gestione dei fenomeni comunicativi in grado di monitorare la società ben prima di attivare procedure drastiche come divieti, soppressioni e cancellature. Ciò offre però lo spunto per un'ulteriore riflessione di carattere metodologico ${ }^{3}$. La censura, tanto quella teatrale quanto quella libraria o giornalistica, svolse certamente il compito di cancellare, vietare o arginare opere e testi; tuttavia essa ha, forse involontariamente, anche conservato. La prassi burocratica dei «rapporti», l'attività di revisione degli uffici di censura che intervenivano sui copioni manoscritti, ha paradossalmente assolto al compito di custodire e collezionare nella loro forma originaria molte opere altrimenti condannate all'oblio. Di molti testi, mai stampati e mai rappresentati, esiste un'unica versione custodita nei registri scrupolosamente curati dai revisori. Proprio i censori ebbero, dunque, il merito di salvare ciò che si erano impegnati a impedire che potesse circolare o essere rappresentato.

Il punto di partenza di questa analisi, che intende concentrarsi su un arco cronologico che va dall'età della Restaurazione ai primi decenni postunitari, coincide, per certi versi, col punto d'arrivo.

Tra il 1865 e il I866 il ministero avviò un censimento delle strutture teatrali presenti sul territorio nazionale; l'incarico, affidato alle prefetture, avrebbe dovuto fornire un quadro esaustivo delle sale presenti in ogni provincia, ciascuna con la rispettiva rilevanza ${ }^{4}$. L'indagine in realtà non era mossa dall'esigenza di ottenere dati sempre più concreti sull'entità del patrimonio culturale e artistico del nuovo Stato, ma più prosaicamente dalla necessità di identificare tutti i contesti in cui si sarebbero potute svolgere rappresentazioni coperte da diritto d'autore. I dati acquisiti in quella fase dal ministero offrono un significativo quadro del fenomeno che interessò tutta la penisola durante la prima metà del secolo. Buona parte delle strutture teatrali era presente già dal Settecento, ma a partire dagli anni '2o la tendenza a costruire nuove sale anche in aree più periferiche o a ristrutturare e ridefinire l'assetto architettonico di edifici già esistenti raggiunse una capillarità tale da non poter essere trascurata dai governi restaurati, attenti ad ogni fattore che potesse incidere sull'amministrazione dell'ordine pubblico. Il proliferare di cantieri a cielo aperto per nuove costruzioni o per ridefinizioni strutturali più adatte ai nuovi tempi e alle nuove tendenze fece affermare all'economista di aziende teatrali Giovanni Valle che era raro trovare città che non avessero «riedificato o ampliato

3. Per un approfondimento sul concetto di performatività della censura elaborato nel 1997 da Judith Butler cfr. J. Butler, Parole che provocano. Per una politica del performativo, Milano, Raffaello Cortina, 2010.

4. Circolare esecutiva del 3 I marzo I866 sui diritti d'autore, in Archivio Centrale dello Stato [d'ora in avanti ACS], ministero dell'Agricoltura, Industria, Commercio, Divisione III, Diritti d'autore e teatri, b. I. 
o rimodernato il loro teatro» 5 . Anche se in diversi momenti il fenomeno interessa l'intero territorio nazionale; il Lombardo-Veneto sembra sensibilizzarsi durante la parentesi francese, mentre nello Stato sabaudo e nel Sud si deve attendere l'età postnapoleonica e in particolare gli anni '2o per assistere ad uno sviluppo dell'edilizia teatrale. Meno discontinua appare invece la diffusione di sale teatrali in Toscana e nei territori pontifici dal Settecento, con un fisiologico rallentamento durante la fase francese. I due terzi dei teatri italiani censiti nel I868, su 942 totali, furono edificati a partire dal I8I5: 195 progettati in una prima fase che arriva fino al I840, altrettanti nei due decenni fino all'Unità, mentre nei primi anni postunitari comparvero altre 198 nuove strutture.

In particolare nel primo periodo attorno al teatro e al suo ruolo sociale si sviluppa un intenso dibattito impegnando i governi degli Stati preunitari che in quella fase si confrontavano con problemi di ordine pubblico; il teatro venne identificato come una sorta di «modello dell'ordine e della buona sociabilità», quasi come una «scuola di virtù» dove il pubblico era controllato e controllabile. In quest'ottica, considerato nella sua funzionalità architettonica, come «edificio in cui si svolgono rappresentazioni o dove si fanno spettacoli», nella prima metà dell'8oo il teatro si carica di una valenza urbana. Il direttore della Scala Angelo Petracchi, in sintonia con Valle, riconosce al teatro un ruolo centrale non solo come «sollievo delle popolazioni», che avrebbero dovuto trovare negli spettacoli «ricreazione e divagazione», ma in particolare come «scuola e ammaestramento» della società ${ }^{6}$. D'altro canto la vigilanza austriaca sulle manifestazioni teatrali e sul termometro dell'esprit public a Milano è una delle costanti della linea politica di Metternich come dimostrano i rapporti informativi destinati al Cancelliere:

Gli spettacoli teatrali che in qualunque altra città non hanno maggiore importanza di quella che si riferisce alla vigilanza sulle pubbliche adunanze ed alle ispezioni di censura, formano nella città di Milano un argomento di tal rilievo da esigere che per parte dell'IR Corte e del Governo vi si presti la più seria attenzione... L'interesse che vi prende il pubblico, non solo di Milano ma quello ben anche delle altre città provinciali non può essere più grande, ond'è che la riuscita degli spettacoli di quel teatro formano soggetto principale dei discorsi e delle discussioni di ogni conversazione anche in riguardevole distanza dalla capitale ${ }^{7}$.

Per i governi preunitari, assillati e quasi ossessionati dalla necessità di un controllo dei ceti urbani, il teatro (più come luogo che come momento artistico) offre un'ottima occasione di sorveglianza sociale; attraverso una meditata riorganizzazione spaziale delle strutture, a quegli edifici viene attribuito un vero e proprio «ruolo educatore», in grado anche di fungere da equilibratore e regolatore dell'ordine

5. G. Valle, Cenni teorico-pratici sulle aziende teatrali, Milano, Società Tipografica dei classici italiani, I823, p. I73.

6. A. Petracchi, Sul reggimento dei pubblici Teatri, idee economiche applicate agli II. E RR. Teatri alla Scala ed alla Canobbiana in Milano, Milano, Ferrario, I82I, pp. 9-I2.

7. Archivio Storico Civico di Milano (Castello Sforzesco) [d'ora in avanti ASCM], m. 56, Cancelleria Vicereale alla Cancelleria Aulica Riunita di Vienna, Monza 5 settembre I8ı8. 
pubblico. Se da un lato le forze di polizia e gli uffici di censura esercitavano un controllo costante sull'attività teatrale, dall'altro la stessa struttura della sala consentiva un'eccellente riproduzione topografica delle gerarchie sociali dell'epoca neutralizzando i rischi di promiscuità tra ceti. Per certi versi andava riproponendosi in una nuova declinazione l'immagine affermatasi in età giacobina di un teatro con funzioni educative, luogo di formazione e di condivisione dello «spirito pubblico» e di «incivilimento».

Nei primi decenni del secolo alcuni osservatori stranieri indicano il teatro come il luogo in cui maggiormente gli italiani manifestavano la loro arretratezza culturale. E questa l'opinione di Madame de Staël che nel ı8ı6 affermava che in Italia il pubblico andava a teatro «non per ascoltare, ma per unirsi ne' palchetti agli amici più familiari e cianciare» ${ }^{8}$; le faceva eco Stendhal evidenziando che le serate alla Scala fossero «serate di conversazione, dove ci si salutava da un palco all'altro e ci si faceva visita come in un salotto»9. Non sono queste le uniche due voci critiche; ad esse va sommata l'opinione formulata già in età napoleonica da Giovanni Pindemonte, che nel suo Discorso sul teatro italiano, poi edito nel I823, stigmatizzava che gli italiani andassero a teatro solo per scherzevole passatempo considerandolo nulla di più che un «luogo di spasso, di bagordo e di distrazione» ${ }^{\circ}$. Tuttavia a queste considerazioni se ne potrebbero aggiungere altre non discordanti, ma che proponevano una prospettiva differente; è il caso di Samuel Morse che rilevava come in Italia negli anni ' 30 i teatri fossero luoghi d'ordine, tutelati e anche incoraggiati dal governo, che lo difendeva «dagli abusi della folla con un gran dispiego di forze dell'ordine». Un dato su cui tutti concordavano era che il teatro italiano si stesse affermando come luogo di sociabilità, affiancandosi ai circoli, salotti, e caffè di matrice settecentesca, ma rispetto a questi col significativo valore aggiunto di poter diffondere «nei cuori degli spettatori la più utile e la più sana morale» ${ }^{\mathrm{II}}$.

La politica teatrale rappresentava dunque una sorta di architrave in grado di coniugare l'esigenza di sicurezza e di ordine pubblico con un più ampio intervento nel settore culturale, fornendo una strategia per canalizzare la tendenza all'associazionismo in una dimensione costruttiva, rivolta a delineare un modello di società controllabile e orientabile attraverso un luogo ben delimitato e rappresentazioni opportunamente selezionate, sempre sotto l'attenta supervisione degli uffici di censura. Va precisato che nelle legislazioni degli Stati preunitari, salvo qualche eccezione, non esisteva una normativa censoria specifica per gli spettacoli

8. A.L. Staël-Holstein, Sulla maniera e l'utilità delle traduzioni, in «Biblioteca Italiana», n. I, I8I6, ora anche in Discussioni e polemiche sul Romanticismo (I816-I826), vol. I, a cura di E. Bellorini, Bari, Laterza, 1943, p. 8.

9. Stendhal, Roma, Napoli, Firenze. Viaggio in Italia da Milano a Reggio Calabria, Roma-Bari, Laterza, 1990, p. 8.

Io. G. Azzaroni, La rivoluzione a teatro, Antinomie del teatro giacobino in Italia, Roma, Bulzoni, 1985, p. 78; cfr. anche C. Sorba, National theater and the age of revolution in Italy, in «Journal of modern italian studies», vol. 17, n. 4, 2012, pp. 400-413.

II. Avviso pubblico del teatro di Modena, I820, in Archivio di Stato di Milano [d'ora in avanti ASM], Spettacoli pubblici, b. 98. 
teatrali e le competenze di supervisione erano definite dai regolamenti delle opere a stampa, vigenti già dal Settecento, ma che tra il I816 e il I823 vennero quasi ovunque ripubblicati o revisionati ${ }^{2}$. La censura teatrale era pertanto regolata da una sorta di «derivazione interpretativa» tratta dai regolamenti generali sulla censura libraria $^{13}$. La pressoché totale assenza di una legislazione teatrale, salvo quella di carattere commerciale, affidava dunque la gestione del sistema, caso per caso, alla contrattazione tra impresari e autorità locali.

L'inscindibile relazione tra teatro, politiche di controllo dell'ordine pubblico e progettazione dell'assetto urbano si manifesta limpidamente soprattutto nel Lombardo-Veneto dove l'attenzione non venne rivolta solo alla Scala ${ }^{\mathrm{I} 4} \mathrm{o}$ alle sale presenti nei grandi centri, ma anche e soprattutto alle periferie, dove si assiste alla comparsa di teatri al di sotto dei mille posti in numerose cittadine di provincia. Nello Stato sabaudo il fenomeno appare più contenuto e lento ad attecchire rispetto ai territori asburgici limitrofi, almeno fino al I82I. Paradossalmente una svolta in questa direzione proviene proprio dal sovrano che si trova a dover gestire l'ordine pubblico nella fase successiva ai moti insurrezionali. Non va infatti dimenticato che proprio il Teatro d'Angennes di Torino fu uno dei palcoscenici da cui partirono i moti del I82I. Con Carlo Felice prende forma, in modo ancora più marcato che altrove, il binomio censura-politica teatrale volto a potenziare il controllo sociale. Il suo impegno in ambito teatrale, che avrà la più eloquente manifestazione a Genova con la struttura a lui intitolata nel 1825 , è contestuale a una ridefinizione degli assetti censori con un progetto, da lui stesso auspicato, di un nuovo regolamento su libri e stampa periodica elaborato proprio tra il 1822 e il I826, nel quale si affermava che la censura fosse diventata «uno degli affari più importanti dello Stato» dopo gli sconvolgimenti europei degli anni precedenti ${ }^{15}$. Sempre nel I823 venne elaborato un sistema censorio per le opere teatrali che rimarrà in vigore fin dopo il $1848^{16}$. In questo complesso quadro non va dimenticato che lo stesso Carlo Felice ha la paternità della riforma che introduce la prima normativa sul diritto d'autore nel I826, intervento che mirava a proteggere gli intellettuali e più in generale tutte le forme di espressione artistica del regno, alimentando negli anni successivi speranze e ambizioni di intellettuali, musicisti ed editori favorevoli ad un moderato liberalismo che superasse la fase restaurativa ${ }^{17}$.

I2. M.I. Palazzolo, I libri il trono l'altare: la censura nell'Italia della Restaurazione, Milano, FrancoAngeli, 2003; N. Gabriele, Modelli comunicativi e ragion di Stato. La politica culturale sabauda tra censura e libertà di stampa (I720-I852), Firenze, Polistampa, 2009.

13. C. Di Stefano, La censura teatrale in Italia (I600-1962), Bologna, Cappelli, I964, p. 49.

I4. Sulla storia amministrativa della Scala cfr. ASCM, Fondo Spettacoli pubblici, mazzi 5, I5, 56, 60.

I5. Archivio di Stato di Torino [d'ora in avanti AST], Materie economiche, Istruzione pubblica, Proprietà letteraria, Revisione libri e stampe, m. I, Progetti di Regie Patenti concernenti alla revisione de' libri e delle stampe, 1826 .

I6. Ibid. m. I; dopo il I848 nel regno sabaudo il Prof. Carlo Facelli, già attivo negli anni precedenti, verrà confermato come censore e supervisore sulle opere teatrali.

17. L.C. Ubertazzi, I Savoia e gli autori, in «Quaderni di Aida», n. 3, 2000, pp. 27-30. 
Rispetto al secolo precedente in cui il ruolo di revisore di opere a stampa, compresi i testi teatrali, era affidato alla figura del bibliotecario, spesso espressione del mondo ecclesiastico, con la Restaurazione la supervisione diventa competenza di funzionari laici di polizia, dipendenti dal ministero degli Interni, impegnati ad arginare ogni tipo di manifestazione contro «l'incivilimento» e la «sana morale». Teatri sociali e teatri civici compaiono anche nelle aree periferiche del regno sabaudo e l'esigenza di centralizzare il controllo affidandolo ad autorità statali provoca conflitti di competenza di matrice giurisdizionalista, ma anche contenziosi tra Stato e famiglie aristocratiche che ancora risultavano proprietarie di sale o strutture teatrali, come nel caso di Cagliari e Asti ${ }^{\mathrm{I} 8}$.

Almeno sul piano puramente normativo il Teatro non sembra risentire dei benefici effetti della fase post-statutaria. Nonostante l'affermazione della libertà di stampa le rappresentazioni continuavano ad essere vincolate all'approvazione delle autorità e dunque a sottostare a una forma di censura preventiva. Nel regno sabaudo il ıo aprile I849 venne istituita una Direzione Generale dei Teatri, alle dipendenze del ministero degli Interni, incaricata di sovrintendere agli spettacoli della capitale; diretto da Massimo d'Azeglio e costituita da esponenti del mondo culturale e politico torinese come Domenico Promis, Pietro de Rossi di Santa Rosa e Alessandro Paravia, questo organismo avrebbe dovuto gestire una fase di adattamento della censura teatrale rendendola «giudiziosa, temperata e in perfetta armonia con le istituzioni politiche» ${ }^{19}$.

Nel 1852 il ministro Galvagno indicava esplicitamente che a guidare l'opera di revisione doveva essere «il principio morale» e non quello politico, inviando un chiaro segnale di impostazione liberale alle autorità competenti sul teatro ${ }^{20}$. La produzione operistica nel regno sabaudo durante il decennio di preparazione all'Unità appare eterogenea e priva di un preciso inquadramento politico e culturale, riflettendo i grandi fermenti della società, le aperture offerte dalla nuova condizione istituzionale e soprattutto la comparsa di nuovi soggetti artistici provenienti dal resto della penisola e dalla Francia alla ricerca di spazi in cui fosse garantita la libertà di espressione.

Nel I856 la Direzione, inadeguata a gestire la mole di spettacoli, venne sostituita con un Ufficio di Censura Teatrale, anch'esso espressione del ministero degli Interni e diretto da Giovanni Sabatini, con la collaborazione di Giacinto Rivelli e del poeta melodrammatico Giovanni Peruzzini. Per facilitare l'attività dei commissari si pubblicarono elenchi di opere ammesse e vietate; il primo elenco del I857 conteneva un «indice» di ben 250 opere vietate ${ }^{21}$. L'imperativo era salvaguardare

I8. Cfr. F. Ruggieri, Storia del teatro civico di Cagliari, Cagliari, Ed. Della Torre, 1993, pp. 69 ss.; A. Giovine, Il Teatro Alfieri di Asti, Bari, Centro studi baresi, I989, p. 5.

I9. C. Di Stefano, La censura teatrale, cit., p. 88.

20. È illuminante il giudizio espresso da Ugo E. Imperatori nel I9I2 sulla svolta nella politica teatrale dello Stato sabaudo che fissava «un criterio direttivo e un limite agli abusi dei revisori»; U.E. Imperatori, Teatri e libertà, in «Nuova Antologia», I6 marzo I9I2.

2I. C. Di Stefano, La censura teatrale, cit., p. 94. 
da scherno e sarcasmo la religione, tutelata dallo Statuto. La più penalizzata, per ragioni di opportunità politica, fu la produzione agiografica su Garibaldi; sulla stessa lunghezza d'onda nel I859 vennero riesaminate e considerate ammissibili alcune produzioni francesi in funzione filonapoleonica, rimesse poi al bando dopo Villafranca. L'ostracismo del filone garibaldino fu confermato anche dopo l'Unità e giustificato con la generica esclusione di «tutti i componimenti nei quali hanno parte personaggi viventi dell'uno e dell'altro sesso $»^{22}$. Furono così respinte anche rappresentazioni che portavano in scena personaggi femminili controversi come Giovanna d'Arco, Anita Garibaldi o Cristina di Belgioioso ${ }^{23}$.

Il fermento teatrale dell'area centro-settentrionale della penisola è di gran lunga superiore a quello del meridione, con l'eccezione di Napoli, dove gli spettacoli erano diretta emanazione della corte. Nel regno borbonico la politica urbanistica rivolta alla costruzione di strutture per pubbliche rappresentazioni è intensa e attentamente pianificata a partire dalla scelta della dislocazione logistica fino al controllo degli spettacoli che vede sovrapporsi intendenti, funzionari locali e autorità religiose, consapevoli delle potenzialità e dei rischi per l'ordine pubblico. Dopo il I848 Ferdinando II intervenne in senso ancora più restrittivo sulla censura libraria e teatrale inasprendo la normativa in vigore dal secolo precedente. Con i decreti del I3 agosto I850 e del 7 aprile I85 il sovrano affidava ai funzionari di polizia la supervisione su spettacoli e rappresentazioni pubbliche, mentre una speciale commissione di docenti universitari ed ecclesiastici aveva il compito di revisionare libri, giornali, ma anche testi e libretti; il censore Giuseppe Bozzo ebbe la competenza specifica sulle opere teatrali. La censura intercettò tragedie e melodrammi come Gomez da Lione di Girolamo Floreno e Zulima di Giuseppe Sapio, ma anche commedie come Pulcinella a Sebastopoli e Il medico magnetizzatore $^{24}$. Divieto assoluto era sanzionato per spettacoli che citassero direttamente o richiamassero alla memoria le figure o le opere di Lutero, Campanella, Voltaire e Gioberti. Il rigore che travolgeva anche spettacoli innocui scatenò lo sdegno di un osservatore come Marco Monnier che condannò senza appello il sistema censorio borbonico: «ma il Paese, questo popolo, anziché proteggerlo, lo perseguita, né mai dispotismo più arbitrario e più puerile oppresse né molestò col pensiero umano, colla ostinazione e le sciocchezze che fece la polizia a Napoli dopo il I848 $\gg^{25}$.

Se la Toscana è forse il territorio che maggiormente si avvicina al modello francese con una crescita progressiva di nuove strutture teatrali che spinse Stendhal ad assimilare Firenze ad una «cittadina francese di terz'ordine quanto a teatri», ciò è dovuto anche all'apprensione che spinse il Granduca a sostenere e ad alimentare capillarmente il fenomeno che interessò in modo significativo anche Livorno

22. ACS, Ministero dell'Interno, Segretariato Generale, Divisione V, bb. I3-I4.

23. Ibid., b. I4.

24. C. Di Stefano, La censura teatrale, cit., p. 49-50.

25. M. Monnier, L'Italie est-elle la terre des morts?, Paris, L. Hacette et C., I860, p. 264. 
e Pisa ${ }^{26}$. Sul piano normativo vigeva sempre la direttiva censoria del I743, ma una Notificazione del 26 dicembre I8I4 aveva ribadito che «nei casi delle licenze per i teatri dovrà sempre precedere la licenza del Presidente del Buon Governo anche per ciò che riguarda la qualità degli spettacoli». L'«Accademico d'ispezione», il funzionario incaricato di sovrintendere alle opere, aveva il potere di interrompere gli spettacoli e procedere ad arresti immediati in caso di rappresentazioni che avessero minato «la conservazione del buon ordine e della pubblica decenza». Le maglie censorie toscane appaiono meno stringenti e vincolanti che altrove, anche se questo aspetto emerge più limpidamente nella prassi che nella normativa. Come nel resto della penisola anche in Toscana le opere di Alfieri furono le più bistrattate. Tuttavia l'ostracismo della censura granducale nei confronti dell'autore sembra concentrarsi più sulle rappresentazioni sceniche che sulla stampa delle sue opere ${ }^{27}$. Nel solo biennio I821-22 vennero respinte La congiura dei pazzi, Don Garzia, Caio Gracco, Bruto I e Suor Virginia. Negli anni successivi, nonostante ripetuti tentativi, non vennero rappresentate neppure Il Misogallo e La Tirannide. La solidità dell'impalcatura istituzionale fondata sull'asse Trono-Altare non lasciava margini di affermazione ad opere ideologicamente in antitesi con lo spirito restaurativo. Il revisore sottolineava che «le opere dell'Alfieri hanno un acerbo e profondo odio contro i monarchi e ogni regolamento sociale di Istituzione Monarchica e anche contro la Religione; ad esempio quando cita la religione cristiana come uno stabilimento politico e quindi le più venerate istituzioni della religione e le misure regolamentari della chiesa come imposture» ${ }^{28}$. La Tirannide continuò ad essere oggetto di sequestri anche dopo il I848 e, nonostante una nuova legge che garantiva una maggiore libertà di stampa, anche negli anni successivi a Felice Le Monnier venne proibito di ristampare le opere di Alfieri. Ancora nel I859, durante i primi mesi di regno di Vittorio Emanuele II, a Laura Bon fu impedito di portare in scena La congiura dei pazzi.

Giovanni Battista Niccolini, noto in tutta la penisola per la carica liberale e gli ideali repubblicani di cui erano intrise le sue tragedie, ebbe con gli uffici di censura un contenzioso costantemente aperto. Una celata simpatia del Granduca nei suoi confronti gli offrì in Toscana maggiori spazi rappresentativi che tuttavia non si rifletterono altrove. In alcuni casi, come per Ludovico il Moro e Arnaldo da Brescia la censura fiorentina dovette però allinearsi a quella austriaca del Lombardo-Veneto, mentre il governo asburgico arrivò anche a richiedere alle autorità l'espulsione del Niccolini dallo Stato toscano ${ }^{29}$.

26. A. Polsi, Possidenti e nuovi ceti urbani: l'élite politica di Pisa nel ventennio postunitario, in «Quaderni storici», XIX, n. 56, 1984, pp. 494-5I5; cfr. anche C. Sorba, Teatri. L'Italia del melodramma nell'età del Risorgimento, Bologna, il Mulino, 20oI, pp. 53-55. Per un riferimento comparatistico con la censura negli altri Stati europei nell'soo cfr. V. Granata, Politica del teatro e teatro della politica. Censura, partiti e opinione pubblica a Parigi nel primo Ottocento, Milano, Unicopli, 2008; R.J. Goldstein, The Frightful Stage: Political Censorship of the Theater in Nineteenth-Century Europe, New York, Berghahn, 2009.

27.C. Di Stefano, La censura teatrale, cit., pp. 65-66.

28. Ibid.

29. Ibid., p. 73. 
Anche nei territori sotto diretto controllo asburgico la censura era stata ripristinata nel i8Is con una serie di indicazioni provenienti direttamente da Vienna che riabilitavano i regolamenti settecenteschi. Qui il rapporto tra censura e politica teatrale fu sapientemente calibrato per evitare che la popolazione percepisse un senso di repressione e di ostracismo verso rappresentazioni e autori; al teatro venne affidata una funzione virtuosa di ordine e di educazione morale. Il prefetto di polizia Carlo Giusto Torresani Lanzfeld riconosceva ai teatri il compito di «correggere il costume, essi non mai presentare dovrebbero se non azioni virtuose». Oltre alle opere di Alfieri e Niccolini, dal Lombardo-Veneto vennero bandite tutte quelle di Victor Hugo e ad attenta supervisione vennero sottoposte anche le produzioni di Foscolo, Manzoni e Pindemonte; anche I Martiri di Donizetti vennero trasformati in Paolina ${ }^{30}$.

In ambito urbanistico i territori delle Legazioni pontificie sembrano allinearsi al quadro nazionale con una significativa crescita di piccoli teatri nelle periferie del regno che si sommavano a quelli già sorti negli anni di dominazione francese. Un caso a sé è rappresentato invece da Roma dove i maggiori teatri erano quelli di matrice settecentesca (Apollo, Argentina, Valle), interessati solo da qualche parziale ristrutturazione e che erano proprietà di famiglie blasonate come i Torlonia o i Capranica, le quali li gestivano privatamente accordandosi con gli impresari ${ }^{31}$.

Nei primi decenni del secolo nello stato pontificio quella che poteva definirsi una «triplice» censura, ecclesiastica, politica e municipale, si accanì nei confronti di ogni forma di pubblica rappresentazione considerata al di fuori dei canoni del «buon costume». Eloquente il rifiuto sistematico nei confronti del valzer, danza ritenuta «oscena» dall'abate Somai, uno dei più attivi revisori teatrali fin dagli anni ' $2 \mathrm{O}^{32}$. A partire dal 1847 la sovrintendenza sui teatri e sulle opere venne affidata alla Magistratura romana e nonostante il tentativo di Pio IX di allinearsi alle politiche degli altri governi in tema di libertà di stampa, dopo il I848 il teatro continuò ad essere oggetto di rigida sorveglianza. Pur mantenendo inalterate le prerogative della censura ecclesiastica, infatti, lo Statuto concesso da Pio IX nel ' 48 aboliva la censura preventiva di competenza governativa e politica su tutte le pubblicazioni a stampa (art. XI); lo stesso metro non venne però applicato alla censura teatrale che continuava a sopravvivere in forma preventiva (art. XII) ${ }^{33}$. A partire dal I850, coinvolgendo anche la componente municipale e attribuendole una parte di competenza sulla censura dei libretti, il governo pontificio intendeva mostrare all'opinione pubblica un'apertura, seppur formale, ad una politica partecipata. Più

30. Un interessante caso di «autocensura» è stato studiato da Philip Gossett in merito alle traduzioni in italiano di opere liriche da rappresentare nella penisola; cfr. P. Gossett, Dive e maestri. L'opera italiana messa in scena, Milano, Il Saggiatore, 20I2, pp. 4I3-416.

3I. G. Radiciotti, Teatro e musica in Roma nel secondo quarto del secolo XIX (I825-I850), in «Atti del Congresso internazionale di scienze storiche», Roma, aprile ı903, vol. VIII, Roma, Tip. Della R. Accademia dei Lincei, 1905, pp. 157-318.

32. C. Di Stefano, La censura teatrale, cit., pp. 52-53.

33. Statuto fondamentale pel governo temporale degli Stati di S. Chiesa, Roma, Camera Apostolica, 1848 . 
tacitamente l'obbiettivo era di addossare una parte di responsabilità di eventuali censure e revisioni, nella fattispecie dei libretti, ad un organismo esterno alla sfera pontificia ${ }^{34}$. Inoltre i libretti passavano alla revisione dell'autorità municipale solo dopo il vaglio della censura ecclesiastica e politica ed esclusivamente per una supervisione di carattere filologico ${ }^{35}$. Tutto era attentamente vagliato, non solo i libretti, ma anche il vestiario, i modelli, i bozzetti scenici e i manifesti. Questa supervisione richiedeva l'esistenza di una solida struttura alla quale era affidato il compito di filtrare ogni elemento che potesse instillare nel pubblico opinioni e idee avverse al modello teocratico che si era imposto nei decenni precedenti ${ }^{36}$. Anche gli abiti di scena erano opportunamente esaminati per evitare che potessero determinarsi particolari abbinamenti in grado di richiamare alla mente il Tricolore ${ }^{37}$. Le relazioni di polizia, in particolare di monsignor Antonio Matteucci, direttore generale di polizia e presidente della Deputazione dei pubblici spettacoli, paiono estremamente efficaci nell'offrire il clima che circondava una pubblica rappresentazione a Roma: «nel gioco dei cavalli si verificò quest'oggi che due buffi pagliacci vestivano i colori dell'italiana demagogia che destarono la simpatia di pochi perversi $\gg^{38}$.

Tra il I852 e il I853 anche Gioacchino Belli rivestì il ruolo di censore teatrale, godendo di grande considerazione a quanto emerge dalla sua fitta corrispondenza col Matteucci ${ }^{39}$. Durante il suo biennio di attività Belli fu chiamato ad esprimere dei pareri di revisione su undici opere ${ }^{40}$; il giudizio sul suo operato è forse già icasticamente contenuto tutto nella secca analisi di uno suoi primi biografi, Domenico Gnoli: «la censura di quei tempi, già ridicola pei suoi eccessi, pareva troppo larga e tollerante al nostro Belli» ${ }^{4}$. Senza voler sottovalutare l'eccesso di zelo del poeta romano, merita di essere rimarcata la scelta del governo di affidare il compito di revisore a una personalità della quale non solo era nota la meticolosità, ma la cui cultura avrebbe anche garantito un più approfondito livello di analisi e di lettura delle opere. E Belli, uomo del suo tempo, si concentrò prevalentemente contro quelle manifestazioni artistiche che in qualche modo riflettevano e propagandavano la nuova sensibilità politica rappresentata dal moderatismo liberale, nella sua ottica ben più pericoloso, perché maggiormente in grado di affascinare frange della società

34. Archivio di Stato di Roma [d'ora in avanti ASR], Direzione Generale di Polizia, Ordine pubblico, Regolamento generale per la Deputazione Pubblici spettacoli.

35. Ibid.

36. Nel I857 per il teatro Metastasio fu stilato un regolamento in undici punti rivolto agli impresari; cfr. ASR, Direzione Generale di Polizia, 6 dicembre 1857.

37. ASR, Direzione Generale di Polizia, Tribunale criminale del Vicariato, 6 dicembre 1857.

38. ASR, Ministero dell'Interno, Teatri, Disposizioni generali, I85I-I852; cfr. anche ibid., Sez. II Spettacoli Pubblici n. 22100-1853 24495del 29 dicembre I857 e n. 2210024496 del 24 dicembre 1857.

39. L. Rivelli, G. Gioacchino Belli «censore» e il suo spirito liberale, in «Rassegna storica del Risorgimento», a. X, 1923, pp. 318-393.

40. I racconti della Principessa di Navarra, Una commedia dopo l'ultima recita, La Calunnia, Marianna, Macbeth, Un bicchier d'acqua, Non più fischi in teatro, Nabucodonosor, Mosè, Viscardello, Torquato Tasso.

4I. D. Gnoli, Il poeta romanesco G.G. Belli e suoi scritti inediti, in «Nuova Antologia», fascicolo III, I feb. I878, p. 496. 
capitolina. La sua posizione e il suo operato sono pienamente conformi alla normativa censoria che condannava in modo totale ogni tipo di rappresentazione satirica contro governi e religione; è netta a questo proposito la distinzione tra il pubblico funzionario e il poeta che proprio nei suoi sonetti aveva fatto del sarcasmo e della satira le armi più taglienti contro l'amministrazione di Gregorio XVI; ma in questo caso l'involuzione conservatrice va addebitata al biennio rivoluzionario $1848-49^{42}$.

Le politiche che ruotano attorno alla proliferazione di sale teatrali nel primo '8oo si propongono dunque di dar forma a un nuovo luogo di cementazione sociale. Il teatro, sempre più «tempio laico» di un pubblico che rispecchia la metamorfosi della società non diventerà però la «macchina pedagogica» teorizzata negli anni '7o del Settecento da Francesco Milizia ${ }^{43}$, né tantomeno istituzione pubblica in grado di sostituirsi alle autorità e alle leggi, com'era auspicato dai governi restaurati. Quello che frequenta le sale è un pubblico «nuovo», che va a teatro per «riconoscersi», contribuendo a consolidare il rapporto tra politica culturale e morfologia urbana che aveva visto emergere, anche se in modo ancora incerto, una borghesia determinata ad assumere un ruolo da protagonista soprattutto nelle città centro-settentrionali44.

Il teatro italiano della prima metà dell'8oo, dunque, non può essere etichettabile solo come un luogo di controllo della «sociabilità». L'idea della sala come luogo di ordine e silenzio venne screditata in particolare dalla prassi del «parterre attivo»: l'abitudine degli spettatori ad interagire tra loro e con gli stessi attori determinerà una sorta di «spettacolarizzazione del pubblico», trasformando il teatro in uno dei luoghi privilegiati per la circolazione delle idee e delle opinioni. Nonostante la crisi del teatro di parola, il moderno teatro d'opera funge da nuovo catalizzatore sociale, a cominciare dalle scenografie, rappresentando una delle più alte forme artistiche capaci di diffondere e radicare i nuovi valori risorgimentali.

Lo scenografo ottocentesco non è più, come lo scenografo barocco, un artista «di tendenza». I nuovi professionisti del teatro romantico, Alessandro Sanquirico a Milano e Francesco Cocchi a Bologna su tutti, ricostruiscono atmosfere rivolte a un pubblico che dallo spettacolo non si attende più meraviglie iperboliche, ma emozioni in grado di coinvolgere tanto la sfera privata quanto quella collettiva e pubblica. Sanquirico in particolare, responsabile unico di tutti gli spettacoli alla Scala tra il I8I7 e il I832, è lo stereotipo di una nuova figura professionale, impegnato in modo totale (firmò oltre 300 scenografie) in una rivoluzione culturale ${ }^{45}$. Superando il modello neoclassico di doverosa fedeltà storica al testo e di rispetto nella ricostruzione degli ambienti, a partire dagli anni '2o Sanquirico è ispirato

42. ASR, Direzione Generale di Polizia, I2 febbraio I854; 20, 21 gennaio I853.

43. Milizia condannava il teatro a palchetti perché incarnava l'egoismo e il culto privato delle classi più agiate, alimentando l'asocialità e la stratificazione sociale; cfr. F. Milizia, Del Teatro, Venezia, Giambatista Pasquali, 1773, p. 92.

44. M. Tafuri, Il luogo teatrale dall'Umanesimo a oggi, in Teatri e scenografie, intr. di L. Squarzina, Milano, Touring club italiano, 1976, p. 35-36.

45. Teatri e scenografie, cit., pp. I2O-I2I. 
da una libertà interpretativa che riflette, e allo stesso tempo cerca di intercettare, le mutate esigenze del pubblico che affolla i teatri, non più solo nobile, ma anche borghese ${ }^{46}$.

Anche l'affermazione sempre più diffusa della nuova «sala all'italiana» con i palchetti che consentivano al pubblico di essere allo stesso tempo spettatore e parte integrante dello spettacolo, potendosi «vedere e sentire tutti ugualmente», contribuisce a plasmare l'immagine di un teatro che, oltre a proporsi come modello di una nuova forma di sociabilità, si impone anche come luogo di «iniziazione democratica» ${ }^{47}$.

Il melodramma in particolare fu il vero e proprio specchio della società ottocentesca, e della sua stratificazione sociale. Se infatti la stampa rappresentò il mezzo di comunicazione e di diffusione delle idee risorgimentali per le classi più colte, il melodramma ebbe il merito di divulgare con forza ancora maggiore, grazie alla commistione di scena, musica e parole, le idee patriottiche. Inoltre, come sottolineato da Carlotta Sorba, tra melodramma e movimento patriottico-nazionale si creò una simbiosi in grado di dar luogo ad una «melodrammatizzazione della politica» $4^{8}$. La realtà politica si impossessa di un registro e di linguaggi propri delle rappresentazioni teatrali, determinando un processo di «drammatizzazione del reale» che influenzerà in maniera decisiva gesti e comportamenti dei protagonisti del movimento nazionale. È comprensibile quindi la centralità che le autorità attribuivano alla censura per arginare le manifestazioni pubbliche che spesso accompagnavano le rappresentazioni teatrali. Il divieto austriaco del febbraio del I848 di «portare Cappelli detti alla calabrese, alla Puritana, all'Ernani» ${ }^{49}$ rifletteva la consapevolezza del potenziale comunicativo dirompente che queste pratiche di ispirazione teatrale avevano sulla popolazione. La proibizione di indossare copricapo «color rossino così detti agli Ernani» si diffuse presto in tutta la penisola offrendo un'immagine ancora più nitida della propaganda indiretta che il teatro era in grado di esercitare a cominciare dagli aspetti cromatici dell'abbigliamento, capaci di creare suggestioni e imitazioni di matrice patriottica.

In questo contesto serve riflettere sul concetto di censura non solo nell'accezione di «supervisione e repressione sulla produzione culturale», ma anche nel suo significato di «critica». Se non ci si limita ad un'idea di censura solo finalizzata ad arginare e prevenire reati, ma anche e soprattutto a correggere comportamenti devianti dalla «virtù civile», essa può essere analizzata con una chiave di lettura che ne allarga il raggio d'azione. Finché le «virtù civili» coincidevano con gli ideali scaturiti dalla Restaurazione, la censura mostrerà i suoi caratteri conservatori e

46. Sulla Scala sono noti i giudizi entusiastici di Lady Morgan e di Stendhal; cfr. Lady Morgan, Italy, London, I824, vol. I, pp. I6-18; Stendhal, Roma, cit., p. 6; cfr. anche B. Spaepen, «Governare per mezzo della Scala», cit., L'Austria e il teatro d'opera a Milano, in «Contemporanea», IV, n. 4, 2003, pp. 593-620.

47. C. Sorba, Teatri, cit., p. 58.

48. C. Sorba, Il I848 e la melodrammatizzazione della politica, in Storia d'Italia. Annali 22. Il Risorgimento, a cura di A.M. Banti e P. Ginsborg, Torino, Einuadi, pp. 48I-508.

49. C. Spellanzon, Storia del Risorgimento e dell'Unità d'Italia, vol. III, Milano, Rizzoli, 1936, p. 620. 
oscurantisti; ma quando la «virtù civile» inizia a identificarsi con gli ideali di «indipendenza» e di «patria», il controllo sulla circolazione del pensiero assumerà un valore nuovo, nel senso che l'obbiettivo non sarà più evitare la diffusione o la rappresentazione di un'idea o di un concetto, ma al contrario fare in modo che questo possa affermarsi nei modi e nelle forme che le classi dirigenti dell'epoca ritenevano più opportune e congeniali.

Se nella Francia orleanista è marcata la volontà del governo di utilizzare l'opera come strumento di comunicazione e di consenso ${ }^{50}$, in Italia più che di condizionamento governativo va riconosciuto il ruolo svolto dalla polizia e dagli uffici di censura. L'entusiasmo che la parola «patria» e le immagini patriottiche suscitano nel pubblico induce le autorità ad un'attenta supervisione sulle opere, specialmente quelle di Verdi che facevano di quei temi la propria ossatura portante. Nel corso degli anni ' 40 l'ascesa della nuova figura dell'editore musicale mette definitivamente in crisi il sistema impresariale e induce gli autori a non scrivere più per un singolo teatro, intuendo le nuove potenzialità di commercializzazione e di diffusione del proprio prodotto artistico. Verdi incarna questa innovativa tipologia di artista intenzionato a proporsi a professionisti ben più recettivi della propria produ$z^{2}$ ione $^{\text {II }}$. L'intenzione dell'autore di trasmettere e divulgare temi politici in forma musicale appare oggi solo sfumata e appena accennata nei libretti, ma questo è l'esito dell'intervento di una censura sempre più preoccupata della capacità operistica di diffondere sentimenti e ideali patriottici ${ }^{{ }^{2}}$. L'ingerenza censoria è rivolta a disinnescare proprio quel potenziale suggestivo contenuto nelle rappresentazioni modificando titoli, scenari originali, località, nomi, producendo talvolta in Verdi un comprensibile sconforto e il timore di un fallimento artistico. Nel settembre del I848, riferendosi a La battaglia di Legnano, egli rivolge le sue perplessità al librettista Salvatore Cammarano: «Caso mai le censure nol permettessero credete voi che si potrà cambiando titolo, località, etc. ritenere tutta o quasi la verseggiatura? $\mathrm{Al}$ momento conviene andare avanti e finirlo così?» $\$ 3$.

La presenza di soggetti plurali che si ritrovano coesi e uniti contro un nemico comune, l'esperienza vissuta in modo collettivo e l'invito all'azione e alla lotta rappresentano i temi ricorrenti nelle opere verdiane contro i quali i censori attiveranno contromisure incisive anche se talvolta non sufficienti. Ernani, Attila, Nabucco,

50. V. Granata, Politica del teatro e teatro della politica, cit., pp. 348-380; cfr. anche J. Fulcher, The Nation's Image. French Grand Opéra as politics and politicized art, Cambridge, Cambridge University Press, 1987.

5I. Cfr. G. Morelli, L'opera nella cultura nazionale italiana, in Storia dell'opera italiana, Torino, EDT, I988, vol. VI; M. Lavagetto, Quei più modesti romanzi. Il libretto nel melodramma di Verdi, Milano, Garzanti, 1979; M. Mila, La giovinezza di Verdi, Torino, Eri, 1974.

52. Il rapporto tra ideali nazionali e opera teatrale è stato recentemente messo in discussione da Axel Körner, che cita come esempio lo scarso successo di pubblico della Battaglia di Legnano a Bologna poche settimane dopo l'Unificazione; cfr. A. Körner, Opera and nation innineteenth-century Italy: conceptual and methodological approaches, in «Journal of modern italian studies», vol. 17, n. 4, 20I2, pp. 393-399.

53. Carteggio Verdi-Cammarano (I843-I852), a cura di C.M. Mossa, Parma, Istituto Nazionale di Studi Verdiani, 200I, p. 5I. 
Giovanna d'Arco, La Battaglia di Legnano richiamano sistematicamente l'idea della patria «bella e perduta»; ma la forza dei testi di alcune rappresentazioni sta nella volontà di sciogliere ogni ambiguità e di fare riferimento non ad una patria generica, ma a quella italiana ${ }^{54}$.

Gli anni 'so si caratterizzano per un inasprimento dell'operato censorio in ambito teatrale, esito degli eventi del biennio 1848-49. Concepiti nei primi decenni del secolo come strumenti dell'ordine e della buona sociabilità «i teatri avevano finito per funzionare da termometro non più solo del gusto culturale medio, ma del grado di effervescenza politica e ogni allusione all'idea di patria [...] era diventata occasione di trascinamento emotivo» ${ }^{55}$. L'impulso patriottico incarnato dai teatri si manifesta esplicitamente addirittura nel cambio del nome: La Scala diventa «Nazionale», il Concordia di Cremona si trasforma in «Teatro patriottico», inducendo il governo austriaco a soluzioni drastiche come la chiusura o la conversione delle strutture in alloggi militari o ricoveri ospedalieri ${ }^{56}$. Placatasi l'effervescenza dei moti e della guerra i teatri riapriranno, ma stavolta con una repulsione del pubblico e delle stesse deputazioni municipali a rendersi complici e immagine stessa della restaurazione austriaca. Queste forme di desistenza civica spinsero, pertanto, i governi a provvedimenti coattivi in cui i dipendenti pubblici venivano costretti ad abbonarsi, richiamando alla memoria la precettazione teatrale tipica dei primi anni della Restaurazione ${ }^{57}$. Il pubblico avrebbe dovuto tornare a rappresentare lo sfondo di un protocollo di ordine e di moderazione, facendo recuperare al teatro il suo ruolo sociale in una cornice d'Ancien régime.

La tendenza a centralizzare la censura accomuna le politiche del decennio preunitario; tuttavia, fatta eccezione per i primi anni, il teatro riconquisterà il suo potenziale patriottico man mano che ci si avvicinerà al biennio 1859-60, anche se in una dimensione più propagandistica volta a creare consenso in previsione della guerra all'Austria.

In età postunitaria, con l'attribuzione ai prefetti (I864) delle competenze sulle rappresentazioni teatrali, si assiste ad una disparità di trattamento tra stampa e teatro, giacché su di esso continuerà a gravare una sorta di censura preventiva, operata proprio dalle prefetture, abolita su libri e giornali.

È significativo che proprio dopo il raggiungimento dell'Unità nazionale la produzione operistica subisca una significativa flessione di cui non è responsabile solo la censura intesa nella sua forma di revisione e supervisione delle rappresentazioni, ma una nuova e più subdola forma di controllo politico che porta i teatri ad essere coinvolti nella spirale delle vicende parlamentari relative alla loro statalizzazione,

54. C. Sorba, «Or sia patria il mio solo pensier». Opera lirica e nazionalismo nell'Italia risorgimentale, in Gli italiani e il Tricolore. Patriottismo, identità nazionale e fratture sociali lungo due secoli di storia, a cura di F. Tarozzi e G. Vecchio, Bologna, il Mulino, I999, pp. I77-I97.

55. C. Sorba, Teatri, cit., p. 212.

56. B. Spaepen, «Governare per mezzo della Scala», cit., pp. 6r7-6r8.

57. Cfr. Il Teatro Grande di Brescia: spazio urbano, forme, istituzioni nella storia di una struttura culturale, Brescia, Teatro Grande, 1985, p. 98. 
alle competenze comunali e alle controversie con gli impresari sugli appalti. Il passaggio di gestione dei grandi teatri allo Stato impose la costituzione di commissioni locali formate dal sindaco, dal prefetto e da membri elettivi individuati tra $\mathrm{i}$ proprietari dei palchetti, che affrontassero le numerose e specifiche questioni fino ad allora gestite dalle varie soprintendenze. In questo modo riemergeva uno degli snodi che aveva caratterizzato la politica teatrale già nella fase preunitaria, anche se in forme e modelli differenti: il rapporto tra classi dirigenti locali e governo ${ }^{58}$. La tendenza a liberalizzare i teatri, emancipandoli da supervisioni e sovvenzioni governative, non offriva garanzie e manteneva una forte carica di ambiguità. Il dibattito era tra chi, considerando il teatro una ricchezza naturale, auspicava patrocini e sovvenzioni governative, e chi, temendo un'ingerenza statale preferiva un teatro povero ma libero 59 . Se da un lato i teatri di Milano, Parma e Napoli continuavano a godere di finanziamenti pubblici, altre realtà ne erano tagliate fuori; stentava ad affermarsi l'idea di un teatro in forma di «industria»; inoltre la nuova normativa censoria sottraeva all'Ufficio centrale di censura il controllo sulle produzioni locali, conservando l'esclusiva supervisione sulle opere da rappresentarsi a livello nazionale ${ }^{60}$.

In una nota dell'II novembre 1862 Rattazzi specificava ai prefetti che rientrava nelle loro facoltà «proibire la rappresentazione di una produzione ancorché permessa dagli Uffici di censura» ${ }^{6}$. La preoccupazione del ministro non sembra discostarsi dall'interpretazione che del teatro si aveva in epoca assolutista, dal momento che invitava le autorità a diffidare da produzioni di scena che «piuttosto che a correggere riescano a travolgere il giudizio e a pervertire il senso morale del popolo» ${ }^{62}$. La funzione del teatro anche nel neonato Stato nazionale continuava ad essere inquadrata in un contesto di «educazione morale» e di tutela del «pubblico costume». Con la limitazione delle competenze dell'Ufficio centrale di censura, dal I864 i prefetti vennero investiti anche del ruolo di «custodi dell'educazione pubblica» $^{63}$.

In una primissima fase, dal I86I al I863, a preoccupare il ministero furono soprattutto opere di «circostanza», produzioni che cercarono di sfruttare la contingenza dell'avvenuta unificazione facendo leva sull'eccitazione popolare. Si trattava di produzioni di scarso livello, ma tanto numerose da mettere in allarme le autorità. Tra il 1859 e il 1863 furono vietate ben 315 opere teatrali, in grande prevalenza a soggetto politico ${ }^{64}$.

58. I. Piazzoni, Spettacolo istituzioni e società nell'Italia postunitaria (I860-I882), Roma, Archivio Guido Izzi, 20oI, p. II.

59. Cfr. Gazzetta di Milano, II maggio I868, Il ministro Broglio e la musica, in «La Nazione», 27 novembre 1876 , Rassegna drammatica.

6o. ACS, MI, Teatri e scuole di ballo, b. I28; cfr. C. Sorba, Teatri, cit., p. 236-239. Il regolamento dell'8 gennaio I860 stabiliva che nessuna rappresentazione teatrale potesse andare in scena senza l'approvazione dell'Ufficio centrale di censura.

6I. ACS, MI, SG, Divisione V, b. I5.

62. Ibid.

63. I. Piazzoni, Spettacolo, cit., pp. 136-138.

64. ASM, Prefettura, b. 628. 
Nel 1862 si era tentato di offrire strumenti certi e calibrati ai revisori e alle sovrintendenze locali stilando le «Massime per la censura teatrale», una sorta di indice statale delle opere da proibire su tutto il territorio nazionale. Si trattò, tuttavia, di un provvedimento di chiaro stampo politico dal momento che le trenta opere vietate avevano un unico filo conduttore: la figura e le imprese di Garibaldi ${ }^{65}$.

Il ruolo preventivo nella censura teatrale divenne oggetto di riflessione per l'opinione pubblica coinvolgendo anche la stampa. «Il Pungolo», già nel I859, e più avanti anche «Il Diritto», «La Riforma» ospitarono interventi che arricchirono il dibattito, facendo emergere una molteplicità di posizioni, dalle tesi più repressive fautrici di una «censura necessaria» a quelle più critiche nei confronti di questo esercizio ministeriale e votate a una maggiore libertà di espressione ${ }^{66}$.

I primi anni di attività prefettizia furono necessariamente di rodaggio, con interventi spesso arbitrari, privi di uniformità sul territorio nazionale e frutto di eccessivo zelo. Appena tre anni dopo, nel I867, il governo Ricasoli dovette ritornare sulla materia specificando con maggior precisione le tipologie su cui concentrare l'attenzione: rappresentazioni nelle quali si fosse attentato alla morale e al pudore o che incitassero all'odio tra classi; casi di palesi offese nei confronti dei Sovrani, delle istituzioni, del Parlamento e delle leggi dello Stato; infine opere che avessero oltraggiato la vita politica degli individui e i principi costituivi della famiglia ${ }^{67}$. I revisori si dimostrano particolarmente sensibili al rispetto della «pubblica morale»: temi come l'adulterio, le nascite illegittime, la seduzione, delitti, suicidi e atrocità varie venivano respinti come «immorali» ${ }^{68}$.

L'intervento ministeriale si rifaceva in gran parte ad istanze censorie registratesi negli anni precedenti offrendo validi strumenti per un'utile e funzionale schematizzazione. In una prima sezione rientravano gli interventi atti a tutelare lo Stato, le sue istituzioni e i suoi rappresentanti politici. La censura si indirizzò dunque verso i primi casi di satira politica rappresentati nel $1863 \mathrm{da}$ Lo scaccomatto di Vittorio Curtis e successivamente da L'amor di patria o la politica al giorno di oggi e I deputati ossia destra, centro e sinistra, commedie accusate di porre «in ridicolo i deputati della nazione» ${ }^{69}$. In questo primo filone rientravano anche speciali proibizioni rivolte a rappresentazioni che avrebbero potuto offrire una distorta interpretazione dell'età risorgimentale e degli esiti dell'Unità; nel 1862 vennero così proibite Il Risorgimento d'Italia, Celeste, Il canapone che offrivano una rilettura delle annessioni del I859. Con la stessa logica il divieto venne rivolto nel 1863 ai drammi storici I briganti

65. Elenco delle produzioni teatrali respinte da oggi in poi, in ACS, MI, SG, Divisione V, b. I4, Massime per la censura teatrale; cfr. anche I. Piazzoni, Spettacolo, cit., pp. I49-I5I.

66. La censura teatrale, in «Il Pungolo», 29-3I ottobre I859; La censura teatrale, in «Il Diritto», II settembre 1872 .

67. ACS, MI, Circolare del 4 aprile I867.

68. ACS, MI, SG, Teatri, b. I4. Sul ruolo pedagogico del teatro si era espresso peraltro anche Mazzini; cfr. G. Mazzini, Filosofia della musica, note di lettura di S. Ragni, Pisa, Domus Mazzinana, 1996.

69. ACS, MI, SG, Teatri, b. I5. 
napoletani e La sottoscrizione per $i$ danneggiati del brigantaggio ${ }^{70}$. A ciò andava ad aggiungersi un particolare veto per spettacoli che portassero in scena crisi interne a stati stranieri, con particolare riferimento a rivoluzioni o insurrezioni di matrice patriottico-nazionale. Venne dunque completamente respinto tutto un filone operistico riconducibile all'insurrezione polacca contro la Russia zarista del 1863 ( $\mathrm{La}$ Polonia, L'insurrezione in Polonia, Donna Polacca, Un saluto dalla Polonia) e alla rivolta dei contadini dell'est contro l'impero asburgico (Gli insorti di Erzegovina) ${ }^{71}$.

La censura fu molto accorta anche in tema di relazioni internazionali, curando in egual misura l'immagine che negli spettacoli veniva fornita della Francia e dell'Austria. Furono proibiti o subirono radicali modifiche Giovanni da Procida, Vespri siciliani, Emma Lionda, Attila farà da sé, Ladislao Telecki e La cacciata degli austriaci da Genova nel $1746^{72}$

Un secondo principio ispiratore dell'azione censoria riguardava la tutela dei valori etici e della pubblica moralità. Per questa ragione vennero bandite opere che portavano in scena omosessualità o prostituzione: è il caso dell'Armando Davvy e dell'Isabella Orsini nel I86I a Torino e due anni dopo del dramma Chi soffre per amore non sente pena, tutte accusate di «immoralità» e «scurrilità»73. Ad analogo destino andò incontro La povera di Maria de Milis in cui una «mezzana» tentava di «indurre alla perdizione una ragazza» ${ }^{74}$. Sul teatro come veicolo di trasmissione di valori e modelli comportamentali si sviluppò un intenso dibattito sulla stampa che coinvolse testate come «La Riforma», «Il Secolo», «La Gazzetta di Milano», «La Nazione» ${ }^{75}$.

Un terzo ambito molto esteso riguardava gli interventi censori rivolti a rappresentazioni che chiamassero in causa personaggi storici o personalità religiose. La complessità dei rapporti economici e diplomatici tra lo Stato italiano e la Santa Sede richiedeva estrema prudenza e l'indirizzo governativo si rivolse a circoscrivere ed arginare focolai di instabilità sociale. Tra il I86I e il ı863 vennero rifiutate numerose opere come Il denaro di San Pietro, Prete Nicola, Le stragi di Perugia I859, Il parroco del villaggio o Caterina la Contadina e Le vittime dei conventi ${ }^{7}$. Dopo il 1870 , con l'inasprimento dei rapporti, l'attenzione si concentrò su Roma dove il prefetto vietò di portare in scena Olimpia Pamphili (1873), Giuliano l'apostata (1875), Gregorio VII e Beatrice Cenci (1878), tutte ritenute offensive nei confronti della figura del pontefice e della religione cristiana ${ }^{77}$.

70. Ibid., buste I4 e I5.

71. ACS, MI, GAB, Prefettura, b. is.

72. ACS, MI, SG, Teatri, bb. I3 e I4.

73. Ibid., bb. I3 e 15 .

74. Ibid., b. I5; cfr. anche I. Piazzoni, Spettacolo, cit., pp. I67-175.

75. Ibid., pp. 208-210.

76. ACS, MI, SG, Teatri, bb. I3 e I5.

77. ACS, MI, GAB, Prefettura, bb. II8 e 258. Il fenomeno non si limitò a Roma, ma interessò anche altri importanti centri della penisola come Torino, dove nel 1863 la prefettura proibì il dramma Le vittime dei conventi; cfr. ACS, MI, SG, Teatri, bb. I4-I5; cfr. anche F. Festa, Teatro Proibito. In scena i tabù di una nazione, Spoleto, Editoria \& Spettacolo, 20II, pp. I22-I35. 
Questo articolato protocollo sovrintese al mondo del teatro nei decenni successivi, fino all'epoca fascista, con qualche sporadica integrazione come quella voluta da Crispi nel i 888 che assegnava ai delegati di Pubblica sicurezza la facoltà di interrompere le rappresentazioni autorizzate dal prefetto per «ragioni di morale o di ordine pubblico» ${ }^{78}$. Con gli anni' 70 si affermò pertanto un modello censorio volto a individuare nel teatro un ruolo di controllo sociale ed educativo per il popolo ricalcato, in un'ottica di ragion di Stato, su quello dei governi preunitari. I numerosi provvedimenti legislativi che tentarono di sanare con politiche uniformatrici situazioni locali divergenti tra loro e la debolezza finanziaria dei comuni quasi sempre proprietari dei teatri e privi di pubbliche sovvenzioni segnarono la crisi dei teatri cittadini, proprio quando si assiste ad una imponente ripresa edilizia con la costruzione di nuove sale in svariati centri. Questo paradosso, una delle tante contraddizioni postunitarie, è il frutto di numerosi investimenti privati per lo più nel Centro-Nord che rispondevano all'esigenza di spettacolarità proveniente in particolare dal crescente ceto medio: il quale, fino ad allora in gran parte escluso dalle sale, non potendosi permettere l'affitto di un palco, allo stesso tempo rifiutava di essere relegato nel loggione con i non abbienti. Queste nuove istanze ispirarono alcuni costruttori che sul finire del secolo tentarono nuove soluzioni architettoniche che avrebbero modificato l'immagine del teatro italiano. 